\title{
Altered dopaminergic pathways and therapeutic effects of intranasal dopamine in two distinct mouse models of autism
}

\author{
Owen Y. Chao', Salil S. Pathak', Hao Zhang ${ }^{1}$, Nathan Dunaway', Jay-Shake Li ${ }^{2}$ Claudia Mattern,
} Susanne Nikolaus ${ }^{5}$, Joseph P. Huston ${ }^{6 *}$ and Yi-Mei Yang ${ }^{1,7^{*}}$ (D)

\begin{abstract}
The dopamine (DA) system has a profound impact on reward-motivated behavior and is critically involved in neurodevelopmental disorders, such as autism spectrum disorder (ASD). Although DA defects are found in autistic patients, it is not well defined how the DA pathways are altered in ASD and whether DA can be utilized as a potential therapeutic agent for ASD. To this end, we employed a phenotypic and a genetic ASD model, i.e., Black and Tan BRachyury $\mathrm{T}^{+}$Itpr3 $3^{\text {tf }} / J$ (BTBR) mice and Fragile X Mental Retardation 1 knockout (Fmr1-KO) mice, respectively. Immunostaining of tyrosine hydroxylase $(\mathrm{TH})$ to mark dopaminergic neurons revealed an overall reduction in the TH expression in the substantia nigra, ventral tegmental area and dorsal striatum of BTBR mice, as compared to C57BL/6 J wild-type ones. In contrast, Fmr1-KO animals did not show such an alteration but displayed abnormal morphology of TH-positive axons in the striatum with higher "complexity" and lower "texture". Both strains exhibited decreased expression of striatal dopamine transporter (DAT) and increased spatial coupling between vesicular glutamate transporter 1 (VGLUT1, a label for glutamatergic terminals) and TH signals, while GABAergic neurons quantified by glutamic acid decarboxylase 67 (GAD67) remained intact. Intranasal administration of DA rescued the deficits in non-selective attention, object-based attention and social approaching of BTBR mice, likely by enhancing the level of TH in the striatum. Application of intranasal DA to Fmr1-KO animals alleviated their impairment of social novelty, in association with reduced striatal TH protein. These results suggest that although the DA system is modified differently in the two ASD models, intranasal treatment with DA effectively rectifies their behavioral phenotypes, which may present a promising therapy for diverse types of ASD.
\end{abstract}

Keywords: Autism, Fragile X syndrome, BTBR, Fmr1, Striatum, Social behavior

\section{Introduction}

Autism spectrum disorder (ASD) is a prevailing neurodevelopmental disorder, primarily diagnosed by a core of symptoms including social impairments, communication difficulties, restricted interests and repetitive behaviors

\footnotetext{
* Correspondence: jph@uni-duesseldorf.de; ymyang@d.umn.edu

${ }^{6}$ Center for Behavioral Neuroscience, Heinrich Heine University of Düsseldorf, Universitaetsstr. 1, 40225 Düsseldorf, Germany

'Department of Biomedical Sciences, University of Minnesota Medical School, 1035 University Drive, Duluth, MN 55812, USA

Full list of author information is available at the end of the article
}

[1]. ASD patients often show cognitive and mental deficits comorbid with other neuropsychiatric disorders, such as attention-deficit/hyperactivity disorder (ADHD), anxiety, and bipolar disorder [2]. A system-level analysis of brain transcriptome has pointed out that the patterns of gene expression in schizophrenia, bipolar disorder and ASD significantly overlap, and that neurons/synapses are susceptible targets of polygenic modulations in all cases [3]. The common genetic variants and phenotypic traits among these disorders indicate shared neuropathology in the cell signaling pathways. 
The dopamine (DA) system is an intriguing candidate. In the brain, tyrosine hydroxylase $(\mathrm{TH})$ catalyzes the hydroxylation of tyrosine to L-DOPA, which is further converted to the modulatory neurotransmitter DA. DA binds to a large family of G-protein coupled receptors that are classified into two subgroups: D1-like (D1 \& D5) and D2-like (D2-D4) receptors. Dopaminergic neurons primarily originate from substantia nigra pars compacta $(\mathrm{SNc})$ and the ventral tegmental area (VTA) in the midbrain. Projections from SNc to the striatum (STR) form the nigrostriatal pathway that is important for controlling voluntary movement. Projections from VTA to the nucleus accumbens (NAc) and the frontal cortex make up the mesocorticolimbic pathway to regulate memory, reward, motivation and emotion [4]. Similar to ADHD [5], bipolar disorder [6] and schizophrenia [7], DA dysfunction is linked to ASD [8]. The STR and the frontal cortex that receive dopaminergic inputs are altered in human ASD [9-11] as well as in animal models with autistic-like behaviors induced by environmental factors [12]. In vivo imaging data demonstrate presynaptic alterations of DA synthesis and DA transporter (DAT) in the striatal and frontal cortical regions [13]. Interestingly, drugs involved in DA actions, such as risperidone, clozapine, haloperidol and methylphenidate, have yielded beneficial effects in ASD patients [14], although none of them acts selectively on the DA system.

To investigate how the dopaminergic pathways are modified and whether application of DA can have a therapeutic effect in ASD, we employed two distinct mouse models for ASD, i.e., Black and Tan BRachyury $\mathrm{T}^{+} \mathrm{Itpr}^{\mathrm{tf}} / \mathrm{J}$ (BTBR) and Fragile X Mental Retardation 1 knockout (Fmr1-KO) mice. The BTBR strain is a phenotypic model for idiopathic ASD, which exhibits impaired sociability, altered ultrasonic vocalization and increased self-grooming behaviors, simulating the main symptoms of human ASD [15]. We also found that BTBR mice display cognitive and emotional abnormalities akin to the psychiatric comorbidity of ASD [16, 17]. In addition, similar neuroanatomical changes between the BTBR model and ASD subpopulations are reported [18, 19]. As to the DA system, BTBR mice show reduced D2, but not D1, receptor-mediated neurotransmission [20]. On the other hand, Fmr1-KO mice are a genetically defined model for Fragile X syndrome (FXS) [21]. FXS is the result of transcriptional silencing of Fmrl gene and lossof-function of its product, FMR protein (FMRP). Given that FXS is a leading inherited form of mental retardation and autism [22], Fmr1-KO animals are widely used for ASD-relevant studies. Characterizations of the Fmr1$\mathrm{KO}$ mouse line have revealed a decreased number of $\mathrm{SNc}$ cells [23], compromised extracellular DA release [24] and disrupted D1 receptor-mediated synaptic transmission in the prefrontal cortex $[25,26]$.
In comparison with wild type (WT) control mice, we used biochemistry, immunohistochemistry and imaging methods to analyze dopaminergic, glutamatergic and GABAergic neurons in the DA pathways in BTBR and Fmr1-KO animals, with respective antibodies against $\mathrm{TH}$, vesicular glutamate transporter 1 (VGLUT1) and glutamic acid decarboxylase 67 (GAD67). Fractal analysis of THpositive axons in the STR was applied to reveal morphological changes of the dopaminergic projections and their spatial relationships with VGLUT1-immunoreactive nerve terminals. Moreover, we evaluated the effects of intranasal application of DA on the behavior and protein expression in BTBR and Fmr1-KO mice. Our results indicate that the DA system is altered differently yet intranasal treatment with DA improves the behavioral deficits in both mouse models, presenting a potential therapy for ASD.

\section{Methods}

\section{Subjects}

BTBR (stock \# 002282), Fmr1-KO (stock \# 003025) and C57BL/6 J (stock \# 000664) mice were purchased from Jackson Laboratory (Bar Harbor, ME) and were housed in a facility accredited by the Association for the Assessment and Accreditation of Laboratory Animal Care. All mice were kept under a 12-h light-dark cycle (light on from 07:00 to 19:00) and reared 3-5 per cage with food and water ad libitum. Male and female mice were maintained in the same room. All procedures were approved by the Institutional Animal Care and Use Committee and the Institutional Biosafety Committee of University of Minnesota, in accordance with the National Institutes of Health guidelines. C57BL/6 J mice served as control because they are the most commonly adopted control for BTBR mice [27], and share the same genetic background with Fmr1-KO mice (https://www.jax.org/strain/ 003025). Unless specified, male mice (2-4 months old) were used for experiments due to the male-dominant prevalence of ASD [28].

\section{Immunohistochemistry}

Mice ( $n=3$ mice/group for each set of experiments) were anaesthetized with ketamine $(100 \mathrm{mg} / \mathrm{kg}$, i.p.) and xylazine $(10 \mathrm{mg} / \mathrm{kg}$, i.p.), and transcardially perfused with phosphate-buffered saline (PBS, $\mathrm{pH} 7.4$ ), followed by $4 \%$ paraformaldehyde. Brains were removed, post-fixed in $4 \%$ paraformaldehyde overnight at $4{ }^{\circ} \mathrm{C}$, then immersed in $30 \%$ sucrose solution and stored at $4{ }^{\circ} \mathrm{C}$ until they sank. $50 \mu \mathrm{m}$-thick coronal sections were made on a microtome (Leica VT1200 S, Buffalo Grove, IL). They were maintained in $0.3 \% \mathrm{H}_{2} \mathrm{O}_{2}$ solution for $10 \mathrm{~min}$, rinsed with PBS and incubated in blocking solution containing $2 \%$ goat serum and $0.2 \%$ Triton-X 100 at $37^{\circ} \mathrm{C}$ for $30 \mathrm{~min}$. Sections were labelled with a rabbit $\mathrm{TH}$ antibody (1:800; Abcam ab112, Cambridge, MA) at $4{ }^{\circ} \mathrm{C}$ 
overnight. After being washed with $0.2 \%$ Triton-X 100 PBS solution, they were incubated in biotinylated antirabbit blocking solution for $2 \mathrm{~h}$ (1:200; Vector Lab. BA1000 , Burlingame, CA). They were then transferred to an $A B C$ reagent (Vector Lab. PK-6100), stained with 3,3'-Diaminobenzidine (Vector Lab. SK-4100), mounted on slides and cover-slipped with Vectashield (Vector Lab. H-5000). To add fluorescence, after incubation in the primary antibody, sections were incubated in goat anti-rabbit Alexa Fluor 555 (1:1000; Invitrogen A21428, Waltham, MA). 3 h later, they were washed in PBS and cover-slipped with Vectashield (Vector Lab. H-1500). Immunostaining procedures for VGLUT1 and GAD67 were the same, except that primary antibodies guinea pig anti-VGLUT1 (1:5000; Millipore AB5905, St Louis, MO) and mouse anti-GAD67 (1:1000; Millipore MAB5406); and secondary antibodies goat anti-guinea pig Alexa 488 (1:1000; Invitrogen A11073) and goat anti-mouse Alexa 555 (1:1000; Invitrogen A32727) were used.

\section{Imaging analysis}

Images were taken with a Zeiss LSM 710 confocal microscope with 20x and 63x oil immersion objectives or a Leica DMi8 light microscope with a 10x lens. Experimenters, who were blind to the design, analyzed the data using NIH ImageJ software (https://imagej.nih.gov/ ij/). A region of interest (ROI) was selected based on a mouse brain atlas [29]. Areas of anti-TH, -VGLUT1 and -GAD67 signals were manually circled and summated with ImageJ. For each brain region, 2-3 images were sequentially taken with an inter-section interval of $200 \mu \mathrm{m}$. For each image, the intensity of ROI was subtracted from its background. The values of ROI intensity were then normalized to the average values of WT controls. Synaptic boutons were defined within the size of 0.2$3 \mu \mathrm{m}^{2}$ of VGLUT1-positive signals. Co-localization of TH- and VGLUT1-positive boutons were identified with overlaps of the two fluorescent signals.

Fractal analysis of neuronal morphology is a validated methodology [30]. Striatal TH-positive axons were imaged with the $63 \mathrm{x}$ lens ( $6 \mathrm{Z}$-planes, $0.35 \mu \mathrm{m}$ steps, field size: $30 \times 30 \mu \mathrm{m}^{2}$, pixel size: $\left.1024 \times 1024\right)$. Images were stacked to $2 \mathrm{D}$ and processed through deconvolution with ImageJ. A plugin FracLac (https://imagej.nih.gov/ij/ plugins/fraclac/FLHelp/Installation.htm) with an implemented box counting method [31] was applied to calculate box-counting dimension $(\mathrm{Db})$ and lacunarity. $\mathrm{Db}$ and lacunarity are fractal dimensional parameters used for quantifying complexity and inhomogeneity of digital spatial patterns, respectively.

\section{Western blotting}

The striatum ( $n=4-6$ mice/group) was dissected bilaterally at $4{ }^{\circ} \mathrm{C}$ and stored at $-80^{\circ} \mathrm{C}$ until processed. As described earlier [32], tissues were homogenized in icecold lysis buffer, centrifuged at $14,000 \mathrm{rpm}$ for $10 \mathrm{~min}$ and the supernatants were collected for determination of protein concentration. Aliquots of protein $(20-30 \mu \mathrm{g})$ were subjected to $10 \%$ SDS-PAGE and transferred to Immun-Blot PVDF Membrane (Bio-Rad, Hercules, CA). Membranes were incubated in 10\% dry milk in PBST solution at room temperature for $1.5 \mathrm{~h}$ and then in primary antibodies at $4{ }^{\circ} \mathrm{C}$ overnight. Subsequently, they were washed with PBST for $30 \mathrm{~min}$ and incubated in secondary anti-mouse (1:5000; Abcam ab205719) and antirabbit (1:5000; Abcam ab205718) antibodies at room temperature for $1.5 \mathrm{~h}$. Following a rinse with PBST for $30 \mathrm{~min}$, they were reacted with enhanced chemiluminescent reagent (GE Healthcare Life Sciences, Buckinghamshire, UK) and imaged with Odyssey Fc Imaging System (LI-COR Biosciences, Lincoln, NE). Blot intensity was quantified using ImageJ and normalized to $\beta$-actin.

Primary antibodies included rabbit anti-TH (1:500; Abcam ab112), guinea pig anti-VGLUT1 (1:1000; Millipore AB5905), mouse anti-GAD67 (1:5000; Millipore MAB5406), mouse anti-DAT (1:1000; NovusBio mAb16, Littleton, $C O)$ and mouse anti- $\beta$-actin (1:5000; SigmaAldrich A5441, St. Louis, MO).

\section{Dopamine administration}

Mice were handled once a day for at least three consecutive days before drug administration. Dopamine hydrochloride (Sigma-Aldrich H8502) was suspended in a viscous castor oil-based formulation (MetP Pharma, Emmetten, Switzerland). It was freshly prepared in a dose of $3 \mathrm{mg} / \mathrm{kg}$ in a volume of $10 \mu \mathrm{l}$ and kept on ice with protection from light. DA or vehicle suspension was applied $5 \mu \mathrm{l}$ per nostril for $\sim 30 \mathrm{~s}$ with a pipette (Eppendorf North America, Hauppauge, NY). 10 min after administration, animals underwent elevated plus maze, open field or object-based attention tests. Because of their respective deficit in social approaching or social novelty $[16,33]$, in the three-chamber test, BTBR mice received DA before the habituation trial, whereas FmrlKO mice received DA before the sociability trial. As BTBR mice have intact recognition of social novelty $[16$, 17], the social novelty trial was excluded here to avoid excessive administration of DA in the same subjects within a short time. The dosage and timing of DA administration were based on a previous study in mice [34]

\section{Behavioral testing}

BTBR mice ( $n=12$; 8 males and 4 females) were tested using a within-subject design to minimize individual differences. Choice of sex was based on our earlier report that both male and female BTBR mice show autistic-like behaviors [17]. Half of the animals were treated with DA 
and the other half with vehicle. The behavioral tests were conducted in the order: elevated plus maze, open field, object-based attention and three-chamber social test, with an inter-test interval of 24-48 h. One week after the final test, the previous DA group received vehicle and vice versa, followed by the same behavioral testing.

Fmr1-KO mice were randomly divided into vehicle ( $n=7$ males) or DA ( $n=9$ males) group and tested using a between-subject design. Choice of sex was based on the location of Fmrl gene on the $\mathrm{X}$ chromosome with male-dominant occurrence [21]. The order of behavioral tests and the inter-test interval were the same as for BTBR mice. Each Fmr1-KO animal was exposed to each test only once.

Behavioral testing was done in a quiet room $(<40 \mathrm{~dB})$ during 10:00-16:00. LED light provided dim illumination $(\sim 50 \mathrm{~lx})$. Male mice were tested before female ones. Apparatus was cleaned with $70 \%$ ethanol between animals to remove pheromones. A camera was connected to a computer for tracking animals and recording videos (Stoelting ANY-maze, Wood Dale, IL).

\section{Elevated plus maze}

This test was used to assess anxiety-like behaviors [35]. The maze has two open arms $(30 \times 5 \mathrm{~cm})$, two closed arms $(30 \times 5 \mathrm{~cm})$ and a central platform $(5 \times 5 \mathrm{~cm})$ and is elevated at $30 \mathrm{~cm}$ height. The subject was placed on the central platform facing the open arms and given $5 \mathrm{~min}$ to travel freely. Entries to and time spent in the center, open and closed arms, and head-dips, were counted.

\section{Open field}

This test was used to assess locomotion and exploration behaviors. The subject was placed in a polyvinyl chloride box $(40 \times 40 \times 30 \mathrm{~cm})$ for $15 \mathrm{~min}$. Distance traveled, thigmotaxis (distance travelled along the walls), duration of grooming, time spent in the center (virtual central square $13.3 \times 13.3 \mathrm{~cm}$ ) and counts/duration of rearing were analyzed for $15 \mathrm{~min}$ in three 5-min bins. Rearing is a sign for non-selective attention [36]. Self-grooming is a measure of repetitive behavior [37]. Thigmotaxis is an index for sensorimotor function and/or anxiety [38, 39]. Time spent in the center indicates the anxiety level [40]. One BTBR mouse was excluded from the analysis due to lack of rearing.

\section{Object-based attention test}

This test was used to assess attention-associated processes and/or short-term working memory [16]. Objects of different materials (plastic or glass), textures (smooth or rough), sizes (diameter 7-9 cm, height $14-17 \mathrm{~cm}$ ) and shapes (column or irregular) were placed in the open field. Objects weighed enough to prevent being displaced by the animal. Assignment of the objects was counterbalanced to minimize a potential bias for their identity or location. Prior to testing, the animal was habituated to the open field without objects. The actual test consisted of a learning trial (5 min) and a test trial $(5 \mathrm{~min})$ without time delay in between. In the learning trial, the animal was introduced to the field which contained two distinct objects. In the test trial, the objects were replaced by a new one and a copy of either of the explored ones in the same location. During the replacement, the animal remained inside the arena. Object exploration was defined as physical contacts with the objects by the animal's nose, head and forepaws, but not by the body or tail. Climbing or sitting aside the objects was not included. Animals that explored the objects for $<10 \mathrm{~s}$ in either trial were excluded from the analysis. Here, the index $=[$ time spent on the novel object - time spent on the old object] / total time spent on both objects, with a positive value representing intact performance.

\section{Three-chamber sociability and social novelty test}

This test was used to assess sociable behavior and recognition of social novelty [41], in a polyvinyl chloride apparatus composed of three chambers $(20 \times 40 \times 30 \mathrm{~cm}$ each) with passages $(5 \times 5 \mathrm{~cm})$ dividing the chambers. The test included three sessions: habituation, sociability and social novelty (9 min each). In the habituation trial, the subject was placed into the middle chamber and allowed to freely explore the whole apparatus. In the sociability trial, a gender- and age-matched WT mouse that had never been contacted by the subject was put underneath a metal grid cup (diameter $10 \mathrm{~cm}$, height 12 $\mathrm{cm}$ ) in one of the side chambers. Another identical cup was put in the opposite side chamber. The locations for placing the stranger mouse and the empty cup were counterbalanced between subjects. In the social novelty trial, another stranger mouse was placed underneath the previously empty cup. The same strangers were used between subjects. Physical contacts around the cups by the subject's nose, head and forelimbs were defined as explorative behaviors. Sociable index $=[$ time for exploring the stranger mouse - time for exploring the empty cup] / total exploration time. Social novelty index $=$ [time for exploring the novel mouse - time for exploring the familiar mouse] / total exploration time. Positive values represent intact sociability and social novelty preference.

\section{Statistics}

Repeated two-way ANOVAs with "within-subject" factors (treatment, interval or object) were used in the analyses of BTBR behaviors. Mixed two-way ANOVAs with a "between-subject" factor (group) and a "within-subject" factor (interval or object) were used in the analyses of Fmr1-KO behaviors. One-way ANOVAs, paired $t$-tests and one-sample $t$-tests were applied when appropriate. 
Independent $t$-tests (if allowed by results of one-way ANOVAs) or Mann-Whitney U tests (in case of lack of homogeneity or normality of variance) were used for analyzing immunohistochemistry and Western blotting data. Data were expressed as mean \pm standard error of mean (SEM). Statistical significance was set as $p<0.05$. All tests were two-tailed tests. For imaging analyses, $n$ denoted the number of samples from 3 mice per group. Otherwise, $n$ represented the number of mice per group. Sample sizes were determined on the basis of previous studies using similar experimental protocols [16, 17, 33].

\section{Results}

\section{Differential alterations of the DA system in BTBR and} Fmr1-KO mice

To investigate whether and how the DA pathways are modified in the ASD models, we labelled dopaminergic neurons with an anti-TH antibody in brain sections taken from age-, sex- and background matched WT,
BTBR and Fmr1-KO mice. TH is a rate-limiting enzyme that converts tyrosine to DA precursor L-DOPA. We first examined the origins of the DA system, i.e. the $\mathrm{SNc}$ and VTA in the midbrain, and their major projections to the dorsal striatum (dSTR) and NAc via the respective nigrostriatal and mesocorticolimbic pathways [4]. Diagrams in Fig. 1a depict the distribution of the soma and axons of dopaminergic neurons identified by $\mathrm{TH}$ labelling in the four nuclei. Higher-magnification images illustrate the specific locations of these neurons within the SNc and VTA (Fig. 1b). By measuring the intensities of anti-TH staining and normalizing them to the average values of the WT group, we found that TH expression in the BTBR brains was significantly reduced in SNc $(\mathrm{U}=6, \mathrm{Z}=2.146, p=0.032)$, VTA $(\mathrm{U}=7, \mathrm{Z}=2.003, p=$ $0.045)$, and dSTR $(\mathrm{U}=6, \mathrm{Z}=2.143, p=0.032$; MannWhitney $U$ test) (Fig. 1c). In contrast, no differences in $\mathrm{TH}$ expression were observed in the brain areas between Fmr1-KO and WT mice ( $p>0.05$; Mann-Whitney U test).

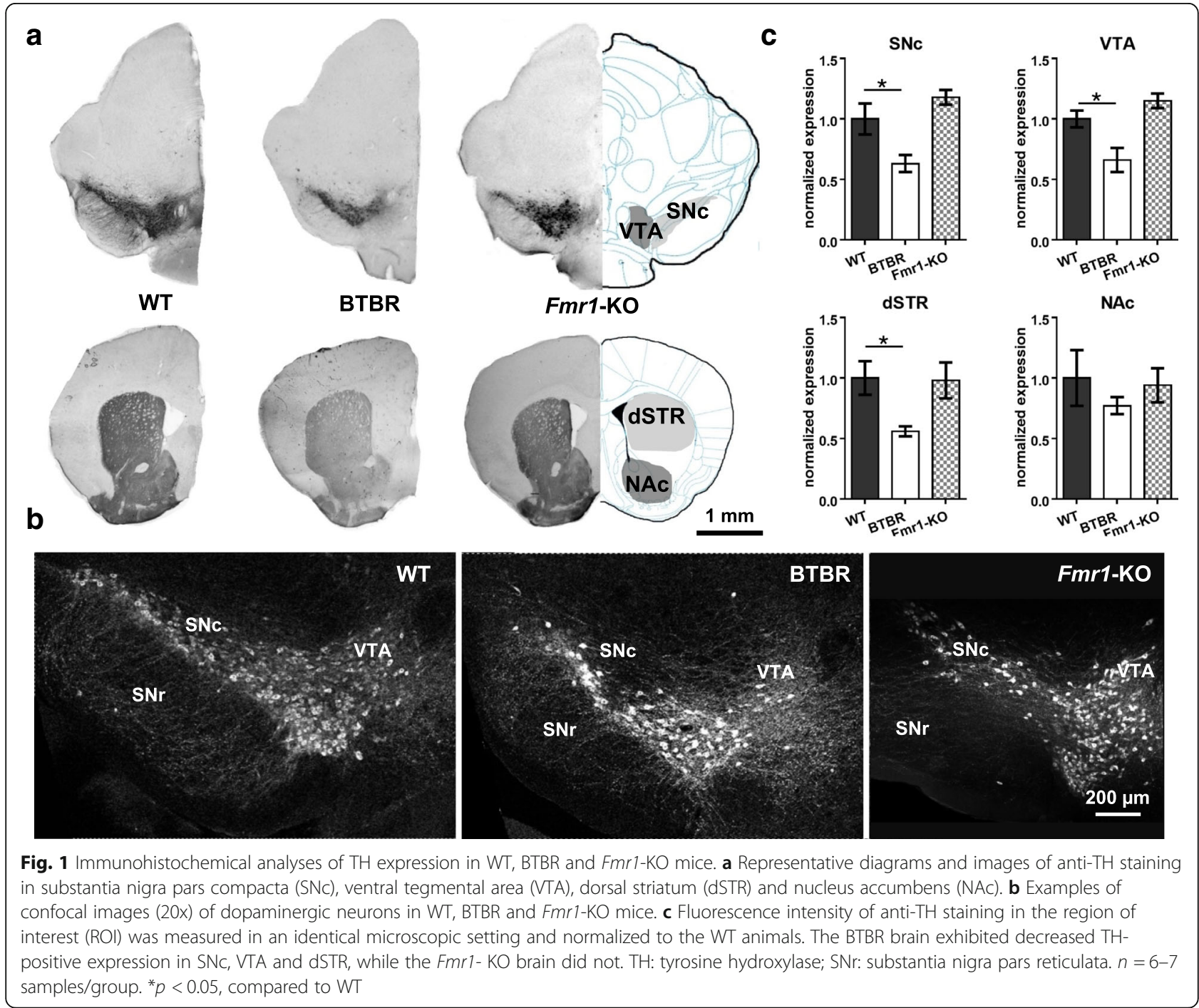


As the STR (mostly dSTR) is a key substrate of the DA outputs and crucially involved in ASD [11], we subsequently focused on the dopaminergic innervations in this region. We noticed a different pattern of $\mathrm{TH}$ positive axons in Fmr1-KO mice as compared to WT and BTBR animals (Fig. 2a). Quantitative assessments of fractal dimensions [30] unraveled significant "group" effects in $\mathrm{Db}\left(\mathrm{F}_{2,20}=6.887, p=0.005\right)$ and lacunarity $\left(\mathrm{F}_{2,20}=14.4, p<0.001\right)$. The Fmr1-KO group had higher $\mathrm{Db}\left(\mathrm{t}_{14}=-2.304, p=0.037\right.$; increased "complexity") and lower lacunarity $\left(\mathrm{t}_{14}=5.126, p<0.001\right.$; decreased "texture"), while BTBR mice displayed no such differences from the WT cohort $(p>0.05)$ (Fig. 2b).

Beside the dopaminergic afferents, the STR receive glutamatergic and GABAergic inputs, and their interactions are essential for the functionality of the basal ganglia. The main excitatory projections come from the cortex and thalamus, which end with terminal boutons that are immunoreactive to VGLUT1 and VGLUT2, respectively $[42,43]$. The two populations of nerve terminals are comparable in the amount and in a similar spatial relation with dopaminergic axons [44]. The inhibitory synapses largely arise from different types of interneurons in the STR and medium spiny neurons in the "direct" (striatonigral) and "indirect" (striatopallidal) pathways [45]. Staining GABAergic neurons with an
anti-GAD67 antibody showed no difference in the intensity of GAD67 labelling among the three groups (WT: $1.00+0.15$, BTBR: $0.97+0.09$, Fmr1-KO: $1.31+0.20$; $p>0.05)$. Likewise, using VGLUT1 as a marker for glutamatergic synapses, we did not detect any significant variance in the overall expression of VGLUT1 (WT: $1.00 \pm 0.11$, BTBR: $1.15 \pm 0.12$, Fmr1-KO: $1.35 \pm 0.24$; $p>0.05)$. However, in the analysis of co-labelled VGLUT1 and TH signals, we uncovered an increased number of VGLUT1-possitive boutons co-localized with the TH-positive axons/synapses in the BTBR $\left(\mathrm{t}_{16}=-\right.$ 3.094, $p=0.007)$ and Fmr1-KO $\left(\mathrm{t}_{16}=-4.309, p=0.001\right)$ STR, compared with the WT brain (Fig. 2c \& d). The size of these boutons did not differ $(p>0.05)$.

Next, we performed Western blotting from striatal homogenates to estimate the quantities of TH, VGLUT1 and GAD67 proteins. In agreement with the immunohistochemical findings (Fig. 1), the total amount of $\mathrm{TH}$ in the STR of BTBR mice was substantially lower than that in the WT brains $(\mathrm{U}=0, \mathrm{Z}=-2.309, p=0.021)$, while VGLUT1 and GAD67 were unaltered $(p>0.05)$ (Fig. 3a $\&$ b). No differences were found in the amount of $\mathrm{TH}$, VGLUT1 and GAD67 between Fmr1-KO and WT groups $(p>0.05)$. Furthermore, we measured DAT, an essential presynaptic protein that modulates DA homeostasis by the reuptake of DA. De novo mutation of DAT

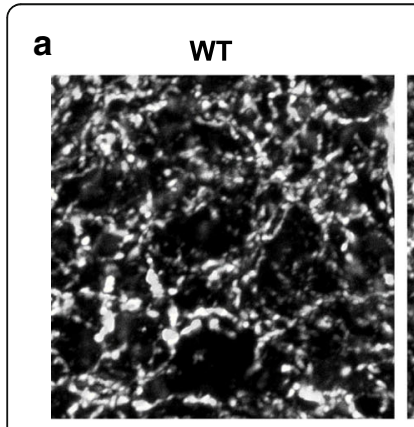

C

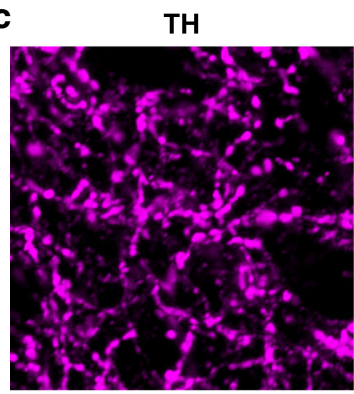

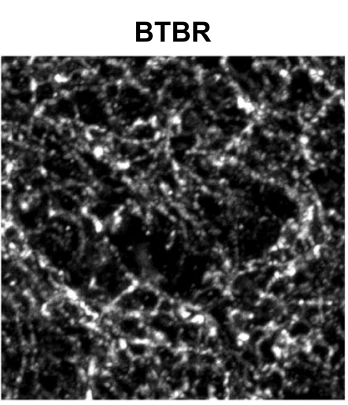

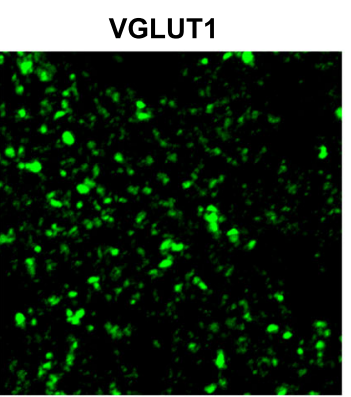

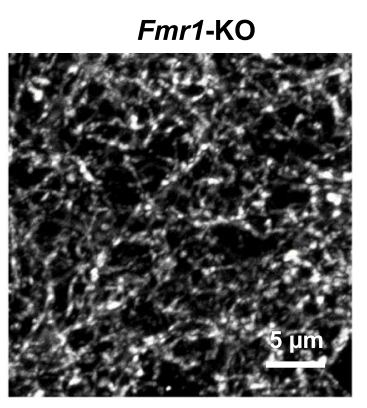

b

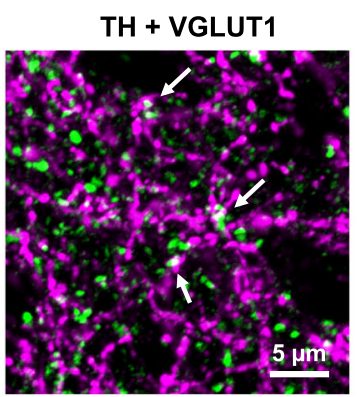

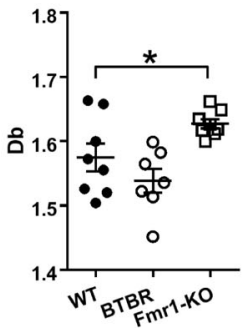

d

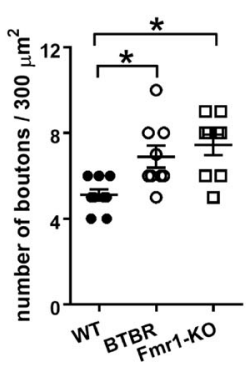

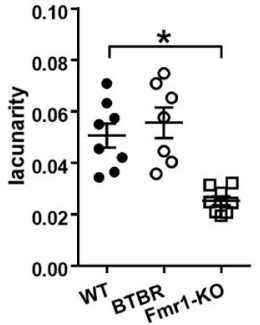

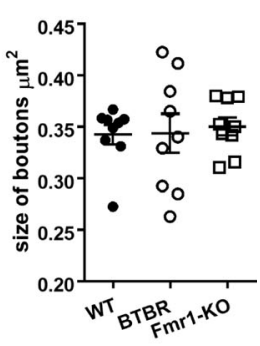

Fig. 2 Fractal analyses of TH-positive axons and measurements of co-labelled TH and VGLUT1 signals in the WT, BTBR and Fmr1-KO striatum. a Representative images of anti-TH staining in the dorsal striatum, taken by a confocal microscope with a $63 \times$ lens, forming a $30 \times 30 \mu \mathrm{m}$ field with 6 z-stacks of $0.35 \mu \mathrm{m}$ steps. $\mathbf{b}$ Fractal dimensions Db of TH-positive axons in the Fmr1-KO striatum was higher, while the value of lacunarity was lower, than those in the WT striatum. c Representative diagrams of anti-TH (magenta), anti-VGLUT1 (green) and their merged image in the WT striatum. Merged dots (white), pointed by arrows, indicate boutons with adjacent TH and VGLUT1 labelling. $\mathbf{d}$ Boutons with the co-labelled signals were counted and their size was measured. The size of the boutons was similar between groups, whereas the number of the boutons was more in BTBR and Fmr1-KO mice than that in WT controls. $n=7-9$ samples/group. ${ }^{*} p<0.05$, compared to WT 


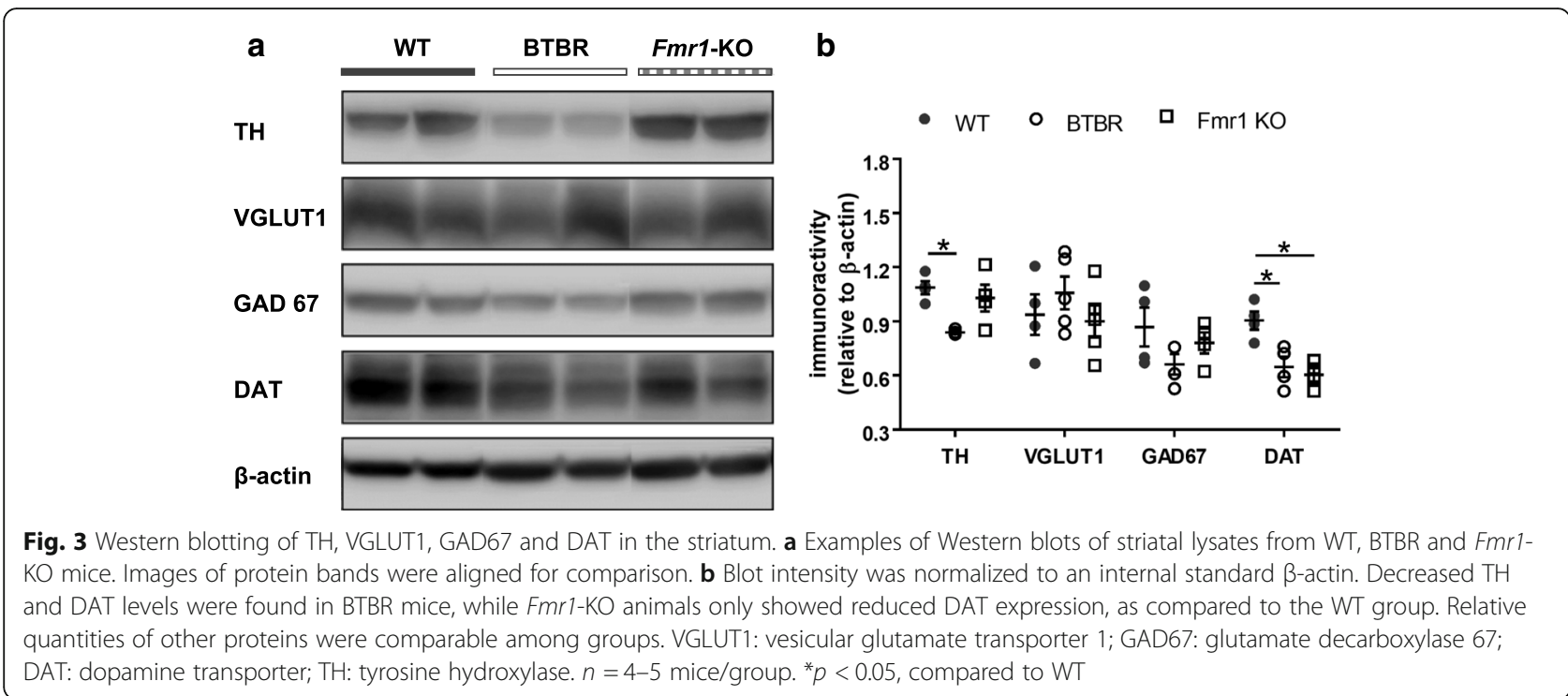

gene is a risk factor for ASD [8]. Interestingly, the DAT levels dropped in both BTBR $(\mathrm{U}=0, \mathrm{Z}=-2.309, p=$ $0.021)$ and Fmr1-KO (U = $0, \mathrm{Z}=-2.309, p=0.021)$ mice, as compared to the WT controls. These changes in the protein expression were confirmed by normalized quantities of TH, VGLUT1, GAD67 and DAT in the BTBR and Fmr1-KO STR relative to the WT cohorts (Table 1).

Taken together, BTBR mice exhibited a global reduction of $\mathrm{TH}$ expression in the cell body and axon projections of dopaminergic neurons in multiple nuclei, suggesting severe detriments along the DA pathways. Fmr1-KO animals did not have such alterations yet showed abnormal morphology of TH-positive axons in the STR. Both strains evidenced more VGLUT1 in close proximity to the $\mathrm{TH}$ signals, indicating an altered regulation of the excitatory inputs by DA. Lastly, the decreased amount of striatal DAT implies deficient DA reuptakes in the two models.

Table 1 Results of Western blots normalized to wild-type (WT) or vehicle (VEH)-treated groups. TH tyrosine hydroxylase, VGLUT1 vesicular glutamate transporter 1, GAD67 glutamic acid decarboxylase 67, DAT dopamine transporter, DA dopamine, KO knockout, N.A. not applicable. ${ }^{*} p<0.05$, compared to WT or VEH groups; Mann-Whitney test. Values are shown as mean \pm SEM

\begin{tabular}{lllll}
\hline & TH & VGLUT1 & GAD67 & DAT \\
\hline WT & $1.00 \pm 0.05$ & $1.00 \pm 0.18$ & $1.00 \pm 0.10$ & $1.00 \pm 0.04$ \\
BTBR & $0.80 \pm 0.04^{*}$ & $1.20 \pm 0.05$ & $0.94 \pm 0.08$ & $0.72 \pm 0.05^{*}$ \\
Fmr1-KO & $1.20 \pm 0.17$ & $1.05 \pm 0.12$ & $1.10 \pm 0.08$ & $0.64 \pm 0.05^{*}$ \\
BTBR + VEH & $1.00 \pm 0.05$ & N.A. & N.A. & $1.00 \pm 0.05$ \\
BTBR + DA & $1.20 \pm 0.07^{*}$ & N.A. & N.A. & $1.00 \pm 0.06$ \\
Fmr1-KO + VEH & $1.00 \pm 0.05$ & N.A. & N.A. & $1.00 \pm 0.05$ \\
Fmr1-KO + DA & $0.71 \pm 0.07^{*}$ & N.A. & N.A. & $1.15 \pm 0.23$ \\
\hline
\end{tabular}

Effects of intranasal DA on striatal protein expression in BTBR and Fmr1-KO mice

Knowing the DA system was dysregulated in the ASD models (Figs. 1, 2, 3), we hypothesized that application of DA might rectify their phenotypes. Because DA cannot pass the blood-brain-barrier due to its polar properties, we administrated DA via the nasal passage [46]. BTBR and Fmr1-KO mice were randomly assigned to vehicle and DA treatments, separately. They were sacrificed 15 min after intranasal administration of either reagent. We quantified the proteins that were altered in their STR with immunoblotting. Compared to the vehicle groups, DA application increased the expression of TH in the BTBR STR $(\mathrm{U}=4, \mathrm{Z}=$ 2.242, $p=0.025$ ) (Fig. 4A \& Table 1), but decreased it in the Fmr1-KO STR (U =0, $\mathrm{Z}=2.739, p=0.006$ ) (Fig. 4b \& Table 1). Given the basal level of TH was lower in BTBR than that in WT mice (Fig. 3), this result implicates that intranasal DA may help rectify the deficiency in the BTBR striatal circuit. In the Fmr1-KO model, the decreased amount of TH following DA delivery may relate to a modulation of FMRP-mediated DA signaling (see Discussion). Although DAT was reduced in both of the ASD strains (Fig. 3), no significant changes were found after DA administration ( $p>0.05$, Fig. $4 \&$ Table 1$)$. Nevertheless, the susceptibility of $\mathrm{TH}$ protein to intranasal application of DA rationalizes the utility of DA for behavioral rescues.

\section{Intranasal delivery of DA alleviates the deficits in non- selective attention, object-based attention and sociability of BTBR mice \\ We performed behavioral assays following intranasal de- livery of vehicle or DA to BTBR mice. In the open field test, we quantified the parameters in three intervals by taking into account confounding factors (anxiety/habitu- ation) that could influence rodent locomotor activity}




\section{a BTBR}
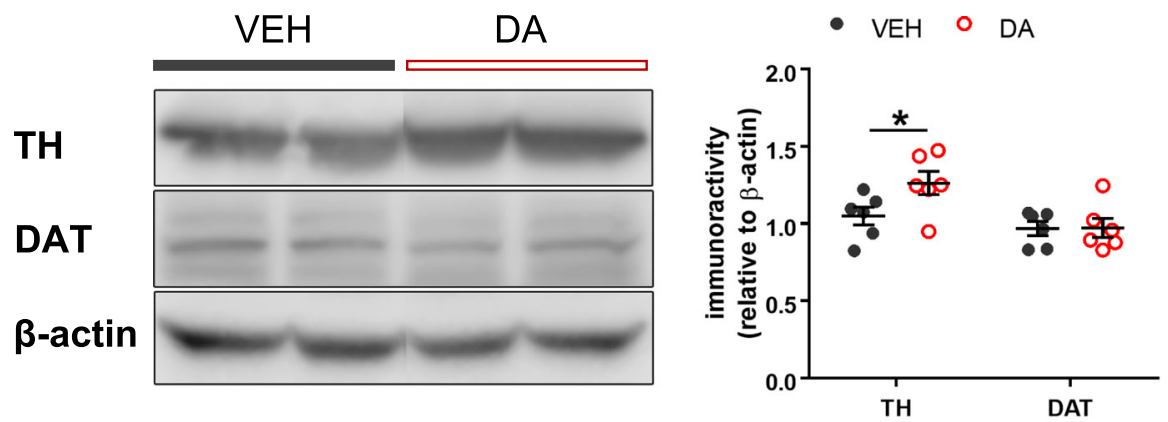

b Fmr1-KO
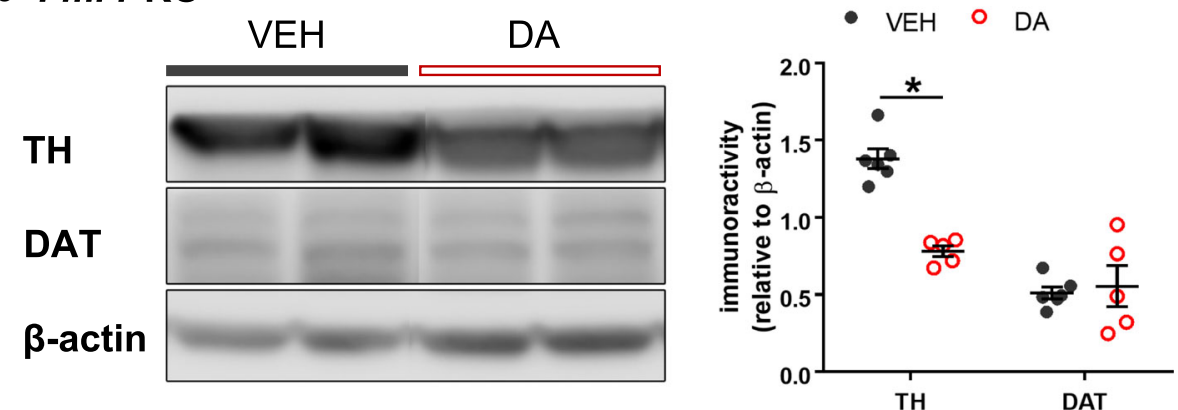

Fig. $4 \mathrm{Immunoblotting} \mathrm{of} \mathrm{TH} \mathrm{and} \mathrm{DAT} \mathrm{in} \mathrm{the} \mathrm{striatum} \mathrm{after} \mathrm{intranasal} \mathrm{delivery} \mathrm{of} \mathrm{vehicle} \mathrm{(VEH)} \mathrm{or} \mathrm{dopamine} \mathrm{(DA).} \mathrm{a} \mathrm{Western} \mathrm{blots} \mathrm{of} \mathrm{striatal}$ lysates from BTBR mice treated with VEH or DA. Increased TH expression was found after DA administration. $\mathbf{b}$ Western blots of striatal lysates from Fmr1-KO mice treated with VEH or DA. Reduced TH level was noticed after DA application. In both cases, DAT protein was unaltered by intranasal DA. TH: tyrosine hydroxylase; DAT: dopamine transporter. $n=5-6$ mice/group. ${ }^{*} p<0.05$, compared to VEH

[47]. Analysis of distance travelled with a repeated twoway ANOVA revealed a significant effect of "interval" $\left(\mathrm{F}_{2,12}=30.519, p<0.001\right)$, but not of "treatment" or "treatment $\mathrm{x}$ interval" ( $p>0.05$; Fig. 5a i). Since the "interval" effect was present, one-way ANOVAs with the within-subject factor "treatment" were applied separately for the periods of $0-5,5-10,10-15 \mathrm{~min}$. No "treatment" differences in the travelling distance were detected at any time intervals $(p>0.05)$. In the analysis of thigmotaxis, we found a significant "interval" effect $\left(F_{2,12}=\right.$ 7.186, $p=0.004$ ), but not "treatment" or "treatment $\mathrm{x}$ interval" effect ( $p>0.05$; Fig. 5a ii). Subsequent examination for each interval uncovered that DA treatment reduced thigmotactic behaviors of BTBR mice in the time bins of $0-5 \min \left(F_{1,11}=6.359, p=0.028\right)$ and $5-10 \mathrm{~min}$ $\left(\mathrm{F}_{1,11}=5.693, p=0.036\right)$. While thigmotaxis is viewed as an index for sensorimotor function and anxiety [38,39], the reduction of thigmotactic behaviors in the DAtreated animals cannot be readily explained by less anxiety because DA did not affect other parameters that reflect the anxiety level, including time spent in the center of the open field and performance on the elevated plus maze (see below). An alternative possibility is that DA may help control excessive thigmotaxic behaviors of BTBR mice [16], by refining their sensory-motor integration. Analysis of self-grooming showed a significant effect of "interval" $\left(\mathrm{F}_{2,22}=8.446, p=0.002\right)$, but not of "treatment" or "treatment $\mathrm{x}$ interval" $(p>0.05$; Fig. 5a iii). In each interval, no "treatment" difference was detected $(p>0.05)$. As to other assessments on time spent in the center and counts of rearing, no effects of "treatment", "interval" or their interaction were observed $(p>$ 0.05; Fig. 5a iv \& v). By contrast, while calculating the average duration of rearing, an indicator for nonselective attention [36], we found a significant effect of "treatment $\mathrm{x}$ interval" $\left(\mathrm{F}_{2,14}=21.463, p<0.001\right)$, but not of "treatment" or "interval" ( $p>0.05$; Fig. 5a vi). Essentially, the animals treated with DA spent more time on rearing than those receiving the vehicle in the first $5 \mathrm{~min}$ $\left(\mathrm{F}_{1,10}=5.146, p=0.047\right)$ but not in other intervals $(p>$ $0.05)$. As BTBR mice show non-selective attention deficits [16], we suggest that intranasal administration of DA improves their non-selective attentional processing without affecting their general locomotion or exploratory activity.

In the object-based attention test, there was no difference in the total time of object exploration between the DA and vehicle-treated groups in either the learning or the test session ( $p>0.05$; Fig. $5 \mathrm{~b} \&$ Table 2$)$. Yet, analysis of the test trial unfolded a significant effect of "treatment $\mathrm{x}$ object" $\left(\mathrm{F}_{1,11}=7.73, p=0.018\right)$, but not of "treatment" or "object" $(p>0.05)$. Paired $t$-tests were 


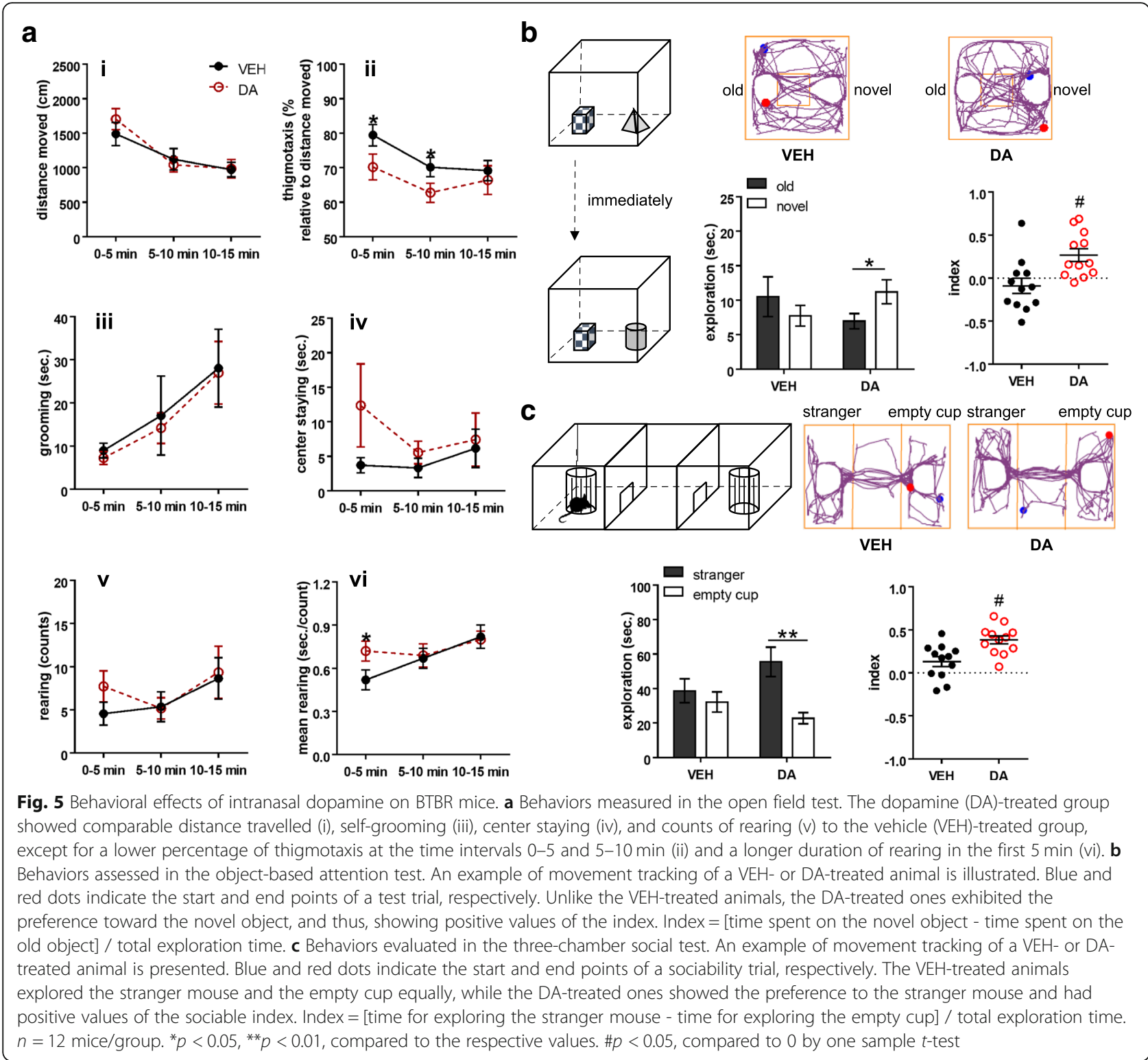

Table 2 Total exploration time (seconds) in the object-based attention test (OBAT) and three-chamber social test. VEH vehicle, DA dopamine, N.A. not applicable. Values are shown as mean \pm SEM

\begin{tabular}{llllll}
\hline \multirow{2}{*}{ OBAT } & \multicolumn{1}{l}{ BTBR } & & & Fmr1-KO & \\
\cline { 2 - 3 } \cline { 5 - 6 } & VEH & DA & & VEH & DA \\
\hline learning & $21.51 \pm 2.29$ & $18.27 \pm 1.22$ & & $24.86 \pm 5.49$ & $26.56 \pm 2.19$ \\
test & $18.16 \pm 2.37$ & $18.54 \pm 3.04$ & & $17.62 \pm 4.66$ & $21.58 \pm 2.93$ \\
Three-chamber & & & & \\
\multicolumn{1}{c}{ sociability } & $70.88 \pm 12.28$ & $78.25 \pm 10.92$ & $62.00 \pm 6.10$ & $62.36 \pm 8.25$ \\
social novelty & N.A. & N.A. & $47.87 \pm 6.42$ & $59.18 \pm 4.87$ \\
\hline
\end{tabular}

then used to compare the exploration time for the old versus the novel object within each treatment. The vehicle-treated BTBR mice explored both objects indiscriminately $(p>0.05)$, consistent with our previous report on their attention/memory deficiency [16]. In contrast, DA-treated animals preferred the novel to the old object $\left(\mathrm{t}_{11}=-2.511, p=0.029\right)$, giving a higher cognitive index $\left(t_{11}=3.588, p=0.004\right)$. The results imply that DA enhances object-based attention and/or shortterm memory of the BTBR model.

In the three-chamber social test, the total exploration time was comparable between the treatments $(p>0.05$; Table 2). Significant effects of "treatment $\mathrm{x}$ object" $\left(\mathrm{F}_{1}\right.$, $10=20.541, p=0.001)$ and "object" $\left(\mathrm{F}_{1,10}=17.834, p=\right.$ $0.002)$, but not of "treatment" $(p>0.05)$, were found in 
the sociability trial. Specifically, the DA-treated animals explored the stranger noticeably more than the empty cup $\left(\mathrm{t}_{10}=4.901, p=0.001\right.$; paired $t$-test; Fig. $\left.5 \mathrm{c}\right)$, while the vehicle-treated mice did not $(p>0.05)$. Accordingly, the DA, but not the vehicle, treatment rendered a positive sociability index $\left(\mathrm{t}_{10}=8.055, p<0.001\right.$; one-sample $t$-test). Considering the characteristics of BTBR mice in their reduced sociability $[15,16]$, this result indicates a beneficial action of intranasal DA on their social impairments. As the BTBR strain has intact social novelty $[15$, 16], we did not continue into the social novelty trial to avoid excessive administration of DA in the same subjects within a short time.

The elevated plus maze test showed no differences in the total distance travelled, entries to and time spent in the center, open and closed arms, and counts of head-dips between the DA and vehicle treatments $(p>0.05$; Table 3$)$. This suggests that DA does not amend the high nonsocial anxiety associated with the BTBR animals [16].

Biological variables, such as sex difference, could contribute to the above observations as both male $(n=8)$ and female $(n=4)$ BTBR mice were included. In the three-chamber social test, for example, conspecific interactions between male subjects and male strangers may have a different innate quality from those among females due to inter-male territoriality and aggression. To test the possibility, we reanalyzed the data by including the males only and found similar results from intranasal DA administration (Table 4), reinforcing the robust effects of DA on the BTBR behaviors.

\section{Intranasal application of DA to Fmr1-KO mice rectifies their defects in object-based attention and social novelty preference}

We executed the same behavioral testing in Fmr1-KO mice after vehicle or DA treatments. In the open field test, a significant effect of "interval" $\left(\mathrm{F}_{2,28}=62.865, p<\right.$ 0.001; mixed two-way ANOVAs), but not of "group" or "group $\mathrm{x}$ interval" $(p>0.05)$, was detected in the analysis of distance travelled (Fig. 6a). Subsequent one-way ANOVAs showed no group difference in the travelling distance at any given time interval $(p>0.05)$. As for thigmotaxis behavior, there was a significant effect of "interval" $\left(\mathrm{F}_{2,28}=100.306, p<0.001\right)$, but not of "group" or "group $\mathrm{x}$ interval". No group differences were found in the three intervals $(p>0.05)$. As for the time spent in the center, there was a significant "interval" $\left(\mathrm{F}_{2,28}=\right.$ $6.895, p=0.004)$, but not "group" or "group $\mathrm{x}$ interval", effect $(p>0.05)$. No group differences were found at any intervals $(p>0.05)$. Assessments on self-grooming and counts and duration of rearing indicated no significant effects of "group", "interval" or their interaction $(p>$ 0.05).

In the object-based attention test, there were no group differences in the total time of object exploration throughout the learning and test sessions $(p>$ 0.05 ; Table 2). In the test trial, there was a significant effect of "object" $\left(\mathrm{F}_{1,8}=22.516, p=0.001\right)$, but not of "group" or "group x object" $(p>0.05)$. The DA group explored the novel object more than the old one $\left(t_{4}=-5.423, p=0.006\right)$, whereas the vehicle group did not $(p>0.05$; Fig. $6 \mathrm{~b})$. Both cohorts had positive object-based attention scores $\left(t_{4}=7.378, p=0.002\right.$ for $\mathrm{DA} ; \mathrm{t}_{4}=3.711, p=0.021$ for vehicle). In light of previous findings on object-recognition impairment mediated by aberrant DA release in Fmr1-KO mice [48], our results indicate intranasal application of DA is an effective avenue for ameliorating the cognitive deficits in the FXS model.

In the three-chamber social test, no group differences were noticed in general explorative behaviors in any of the sessions $(p>0.05$; Table 2$)$. In the sociability trial, there was a significant effect of "object" $\left(\mathrm{F}_{1,13}=73.735, p<0.001\right)$, but not of "group" or their interaction $(p>0.05)$. Both vehicle and DA groups explored the stranger mouse more than the empty

Table 3 Behavioral assessments in the elevated plus maze test. VEH vehicle, DA dopamine. Values are shown as mean \pm SEM

\begin{tabular}{|c|c|c|c|c|}
\hline & \multicolumn{2}{|l|}{ BTBR } & \multicolumn{2}{|l|}{ Fmrl-KO } \\
\hline & VEH & DA & VEH & DA \\
\hline distance $(\mathrm{cm})$ & $816.07 \pm 57.81$ & $965.20 \pm 112.64$ & $1194.93 \pm 126.64$ & $1091.41 \pm 76.38$ \\
\hline \multicolumn{5}{|l|}{ entry (counts) } \\
\hline center & $34.58 \pm 3.06$ & $36.42 \pm 4.18$ & $37.29 \pm 2.29$ & $36.67 \pm 3.02$ \\
\hline open & $13.08 \pm 1.56$ & $12.92 \pm 1.61$ & $14.29 \pm 1.91$ & $15.22 \pm 1.98$ \\
\hline closed & $22.92 \pm 1.64$ & $25.50 \pm 3.02$ & $26.71 \pm 1.66$ & $25.00 \pm 1.59$ \\
\hline \multicolumn{5}{|l|}{ time spent (seconds) } \\
\hline center & $48.88 \pm 6.77$ & $43.01 \pm 4.92$ & $71.37 \pm 4.80$ & $68.18 \pm 7.86$ \\
\hline open & $32.78 \pm 6.29$ & $37.67 \pm 4.99$ & $52.89 \pm 7.88$ & $52.91 \pm 9.39$ \\
\hline closed & $218.33 \pm 11.00$ & $219.32 \pm 9.15$ & $175.71 \pm 8.19$ & $178.90 \pm 10.16$ \\
\hline head-dips (seconds) & $7.19 \pm 2.32$ & $7.74 \pm 1.10$ & $24.83 \pm 2.54$ & $30.47 \pm 4.90$ \\
\hline
\end{tabular}


Table 4 Behaviors of male BTBR mice treated with vehicle (VEH) or dopamine (DA). ${ }^{*} p<0.05$, paired $t$-tests compared to VEH. \#p $<$ 0.05 , paired $t$-tests compared to the empty cup exploration within the same group. $+p<0.05$, one-sample $t$-tests compared to 0 . Values are shown as mean \pm SEM. Note: in the object-based attention test, although the preference for the novel over old object was moderate $(p=0.063)$, the object-recognition index of the DA-treated group remained positive

\begin{tabular}{|c|c|c|}
\hline Elevated plus maze & VEH & DA \\
\hline distance $(\mathrm{cm})$ & $863.63 \pm 78.81$ & $1036.18 \pm 114.71$ \\
\hline center entry (counts) & $36.00 \pm 4.41$ & $38.75 \pm 4.34$ \\
\hline open-arms entry (counts) & $14.38 \pm 2.17$ & $13.75 \pm 2.02$ \\
\hline closed-arms entry (counts) & $23.38 \pm 2.01$ & $27.00 \pm 2.56 *$ \\
\hline center time (seconds) & $51.05 \pm 9.31$ & $46.60 \pm 5.74$ \\
\hline open-arms time (seconds) & $42.10 \pm 7.07$ & $42.23 \pm 6.67$ \\
\hline closed-arms time (seconds) & $206.85 \pm 14.32$ & $211.19 \pm 11.35$ \\
\hline head-dips (seconds) & $8.28 \pm 3.02$ & $8.85 \pm 1.11$ \\
\hline \multicolumn{3}{|l|}{ Open field } \\
\hline distance $0-5 \min (\mathrm{cm})$ & $1451.73 \pm 175.70$ & $1831.94 \pm 122.04$ \\
\hline distance $5-10 \min (\mathrm{cm})$ & $1137.80 \pm 233.01$ & $1184.25 \pm 106.78$ \\
\hline distance $10-15 \min (\mathrm{cm})$ & $980.48 \pm 158.01$ & $1148.89 \pm 155.66$ \\
\hline thigmotaxis $0-5 \min$ (\% by distance) & $76.99 \pm 3.95$ & $64.68 \pm 4.33 *$ \\
\hline thigmotaxis $5-10 \mathrm{~min}$ (\% by distance) & $66.20 \pm 2.98$ & $58.51 \pm 2.97$ \\
\hline thigmotaxis $10-15 \min$ (\% by distance) & $67.12 \pm 4.13$ & $63.13 \pm 5.42$ \\
\hline grooming $0-5$ min (seconds) & $9.33 \pm 2.51$ & $6.43 \pm 1.85$ \\
\hline grooming 5-10 min (seconds) & $21.09 \pm 13.57$ & $17.90 \pm 4.83$ \\
\hline grooming $10-15$ min (seconds) & $31.64 \pm 12.88$ & $30.43 \pm 10.10$ \\
\hline center time $0-5 \min$ (seconds) & $4.00 \pm 1.60$ & $7.94 \pm 2.99$ \\
\hline center time 5-10 min (seconds) & $4.45 \pm 2.00$ & $7.46 \pm 2.18$ \\
\hline center time $10-15$ min (seconds) & $8.04 \pm 4.04$ & $10.13 \pm 5.61$ \\
\hline rearing $0-5$ min (counts) & $6.00 \pm 1.85$ & $8.13 \pm 1.67$ \\
\hline rearing 5-10 min (counts) & $7.00 \pm 2.54$ & $6.00 \pm 1.41$ \\
\hline rearing $10-15$ min (counts) & $10.71 \pm 3.61$ & $11.88 \pm 3.77$ \\
\hline rearing $0-5$ min (seconds/count) & $0.61 \pm 0.08$ & $0.77 \pm 0.07$ \\
\hline rearing 5-10 min (seconds/count) & $0.65 \pm 0.10$ & $0.69 \pm 0.10$ \\
\hline rearing $10-15 \mathrm{~min}$ (seconds/count) & $0.74 \pm 0.15$ & $0.81 \pm 0.08$ \\
\hline \multicolumn{3}{|l|}{ Object-based attention } \\
\hline total exploration (seconds)_learning & $22.06 \pm 3.40$ & $20.40 \pm 1.12$ \\
\hline old object (seconds)_test & $10.18 \pm 2.78$ & $6.15 \pm 1.11$ \\
\hline novel object (seconds)_test & $8.61 \pm 1.54$ & $13.90 \pm 3.77$ \\
\hline index & $-0.05 \pm 0.12$ & $0.31 \pm 0.10 \dagger$ \\
\hline total exploration (seconds)_test & $18.79 \pm 3.24$ & $20.05 \pm 4.31$ \\
\hline \multicolumn{3}{|l|}{ Three-chamber } \\
\hline stranger exploration (seconds) & $45.90 \pm 8.23$ & $60.69 \pm 9.07 \#$ \\
\hline empty cup exploration (seconds) & $37.43 \pm 7.21$ & $25.88 \pm 3.81$ \\
\hline index & $0.12 \pm 0.06$ & $0.39 \pm 0.06 \dagger$ \\
\hline total exploration (seconds) & $83.33 \pm 14.64$ & $86.56 \pm 11.81$ \\
\hline
\end{tabular}

$\operatorname{cup}\left(\mathrm{t}_{6}=5.85, p=0.001 ; \mathrm{t}_{7}=6.493, p<0.001\right.$, respectively) with equally positive sociability indexes $\left(\mathrm{t}_{6}=7.571, \quad p<0.001 ; \quad \mathrm{t}_{7}=12.666, \quad p<0.001\right.$, respectively; Fig. 6c). In the social novelty trial, significant effects of "object" $\left(\mathrm{F}_{1,13}=9.375, p=0.009\right)$ and "group $\mathrm{x}$ object" $\left(\mathrm{F}_{1,13}=11.313, p=0.005\right)$, but 

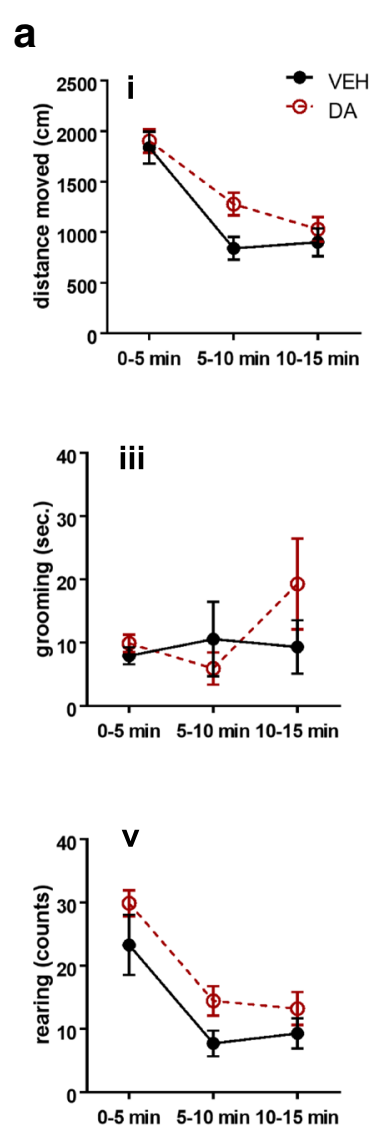

b
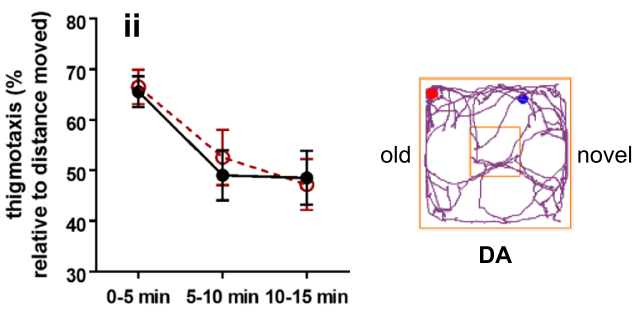

C
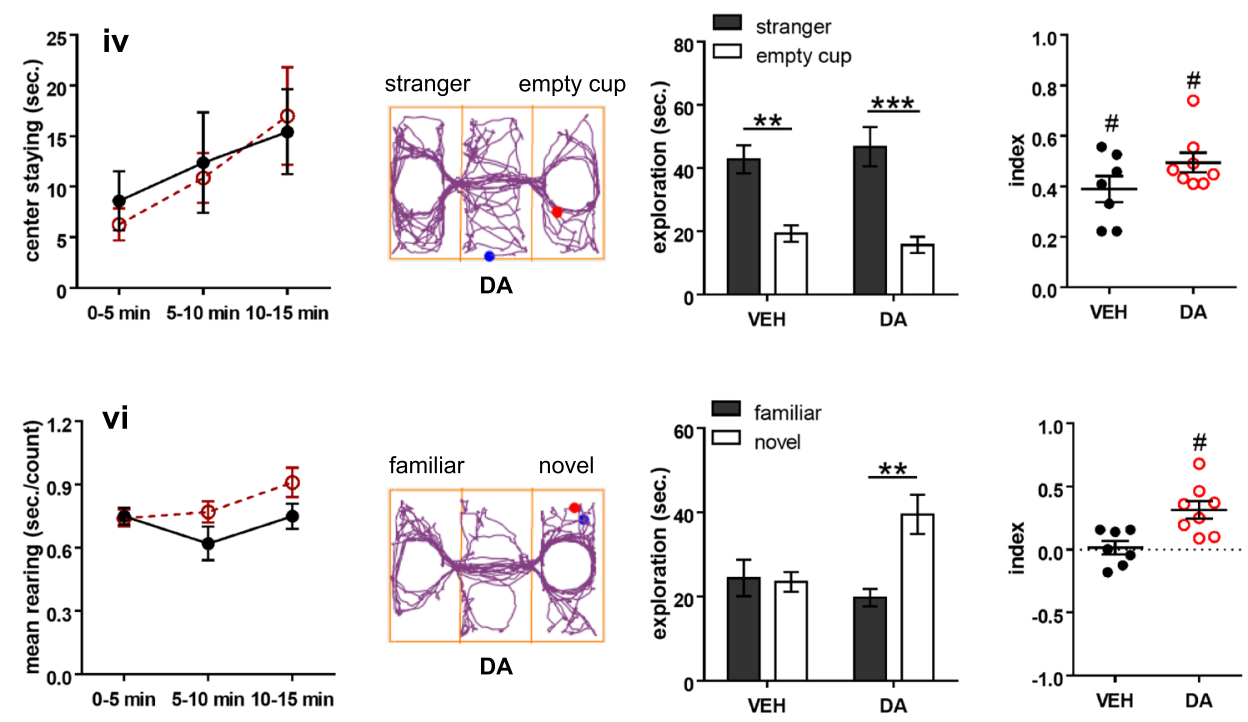

Fig. 6 Behavioral effects of intranasal dopamine on Fmr1-KO mice. a Behaviors measured in the open field test. The dopamine (DA)-treated group showed comparable distance travelled (i), thigmotaxis (ii), self-grooming (iii), center staying (iv), counts of rearing (v), and average duration of rearing (vi) to the vehicle (VEH)-treated group. $\mathbf{b}$ Behaviors assessed in the object-based attention test. An example of movement tracking of a DA-treated animal is shown. Blue and red dots indicate the start and end points of a test trial, respectively. The DA-treated animals exhibited the preference toward the novel object, although both groups had positive values of the index. Index = [time spent on the novel object - time spent on the old object] / total exploration time. c Behaviors evaluated in the three-chamber social test. Examples of movement tracking of a DAtreated animal in a sociability and a social novelty trial are displayed. Blue and red dots indicate the start and end points of each trial, respectively. In the sociability trial (upper panels), both groups displayed social preference toward the stranger mouse and had positive values of the sociable index. Index = [time for exploring the stranger mouse - time for exploring the empty cup] / total exploration time. In the social novelty trial (bottom panels), the DA-treated group explored the novel stranger more than the familiar one, whereas the VEH-treated group did not. The DA-treated group thus had higher values of the social novelty index. Index = [time for exploring the novel mouse - time for exploring the familiar mouse] / total exploration time. $n=7-9$ mice/group (2-4 mice were excluded in the object-based attention test due to $<10 \mathrm{~s}$ exploration). ${ }^{* *} p<0.01,{ }^{* * *} p<0.001$, compared to the respective values. $\# p<0.05$, compared to 0 by one sample $t$-test

not of "group" ( $p>0.05)$, were found. The DA-treated animals explored the novel stranger more than the familiar one $\left(\mathrm{t}_{7}=-3.756, p=0.007\right)$, but the vehicle-treated group did not $(p>0.05)$. Thereby, the DA treatment elevated the social novelty index $\left(t_{7}=-4.45, p=0.003\right)$, however the vehicle failed to do so $(p>0.05$; Fig. $6 \mathrm{c})$. Knowing that Fmr1-KO animals have normal social approaching but atypical social novelty preference [33, 49], we suggest that intranasal DA particularly alleviates the impaired social novelty in the autistic-like Fmr1-KO model.

In the elevated plus maze test, there were no group differences in the behavioral measurements $(p>0.05$; Table 3), indicating a minimal effect of DA on the anxiety level of Fmr1-KO mice.

\section{Discussion}

In this comparative study, we have unraveled distinct alterations and common phenotypes in the DA pathways of two widely adopted mouse models for ASD. BTBR mice showed a hypofunction of the DA system, as indicated by the low expression of TH in several DA centers (Figs. 1, 2, 3), in line with previous studies showing compromised DA-mediated responses in these mice [20]. Moreover, they exhibited decreased motivation for social and food rewards in operant conditioning tasks [50] and less social conditioned place preference [51], which reinforces the perspective of dysfunctional DA system in this model. As $\mathrm{TH}$ is an enzyme for synthesis of both DA and norepinephrine, future studies are required to 
differentiate their roles in the BTBR brain. For Fmr1-KO mice, the TH level did not change significantly (Fig. 3), largely consistent with other reports [23, 24, 49]. Yet, fractal analysis revealed unusual arborization of $\mathrm{TH}$ positive axons in their STR (Fig. 2), strengthening an essential role of FMRP in axon formation [52]. For instance, a loss of FMRP homologue dFMR1 in Drosophila generated aberrant extensions and branches of axons $[53,54]$, while overexpression of dFMR1 led to abridged axonal arbors [55]. In cultured rat cortical neurons, FMRP overexpression attenuated the axon complexity [56]. Furthermore, the axon integrity was altered in the cortex of Fmr1-KO mice [57] and in the dSTR of FXS patients [58]. Despite of the controversy [59], FMRP also plays a role in the development of axon myelination [60, 61]. Whether defective myelination could contribute to the abnormal morphology of TH-positive axons in the Fmr1-KO STR remains unclear. The differences in $\mathrm{TH}$ expression between BTBR and Fmr1-KO mice could relate to their individual genetic background as well.

Co-labeling VGLUT1 with $\mathrm{TH}$ showed an increased number of VGLUT1-containing nerve terminals in close spatial relationship with TH-positive axons, indicating an enhanced interaction between the cortical afferents and released DA in the STR of the two ASD mouse lines (Fig. 2). The molecular underpinnings of DA modulation are complex, for example, depending on the subtypes of DA receptors [62]. Whether DA facilitates or attenuates glutamatergic neurotransmission will be subject to further investigations. Another commonality between the two models was the downregulation of striatal DAT (Fig. 3). DAT is critical for maintaining DA homeostasis by recycling DA from the synaptic cleft to the cytosol. Whole-exome sequencing has identified a DAT mutation in ASD families [8]. Transgenic mice with DAT deficiency showed hyperactivity [63]. Administration of amphetamine, which causes DAT-mediated DA efflux, alleviated self-grooming in BTBR mice [64] and facilitated object recognition in Fmr1-KO mice [48]. More studies are needed to elucidate the mechanisms and consequences of the DAT defect.

The causes for the protein regulation by intranasal DA in the ASD models may be diverse. The bidirectional modulation of the quantity of $\mathrm{TH}$ in the BTBR and Fmr1-KO brain (Fig. 4) likely depends on the different changes in their endogenous DA system (Fig. 3). DA administration in BTBR mice may increase the extracellular DA concentration and DA availability [65], which presumably increases the TH activity in the STR [66]. In Fmr1-KO mice, a lack of FMRP may play a role in reducing the level of $\mathrm{TH}$, considering the interaction between FMRP and DA signaling $[25,67]$. This possibility is supported by an observation that intranasal DA did not change $\mathrm{TH}$ protein in normal rats [68]. As to DAT, increased extracellular DA could affect its binding activity [69]. In spite of the evidence for striatal DAT deficiency in the two strains (Fig. 3), intranasal delivery of DA did not restore DAT expression (Fig. 4).

Intranasal application of DA efficiently rescued the cognitive and social deficits of the BTBR (Fig. 5) and Fmr1-KO (Fig. 6) models. It should be noted that the behavioral assessments of these functions can be confounded by other factors. For instance, hyper- or hypolocomotor activity may influence animals' performance in the object-based attention test and three-chamber social test. However, this unlikely compromised the effects of DA on cognition and social interaction as the overall motor and exploratory behaviors were comparable between the DA and vehicle groups (Figs. 5 \& 6). In rats treated with intranasal DA, an elevated concentration of DA was found in the cerebrospinal fluid and in the brain [70], including the dSTR and NAc [65]. Moreover, intranasal DA had antidepressant-like effects [71], attenuated fear responses [72], compensated behavioral asymmetries in a Parkinsonism model [73], and alleviated cognitive deficits in aged rats [74] as well as in animal models for schizophrenia [75] and ADHD [76]. Therefore, the behavioral rescues by intranasal DA likely stem from brain-wide actions. Here, we focused on the STR because it is a key neuronal correlate to social behaviors [77] and a disparate fronto-striatal circuit has been specified in ASD patients [78, 79]. Mice with deletion of ASD-relevant genes, e.g., Shank3, displayed social impairments, along with decreased corticostriatal neurotransmission, increased morphological complexity of medium spiny neurons, and reduced glutamate receptors in the STR [77]. Although we (Fig. 3) and others did not note dramatic changes in $\mathrm{TH}$ expression in Fmr1-KO animals [23, 24], DA release and uptake were blunted in their STR [24]. The syndromic ASD model, BTBR mice with polygenetic mutations [27], presented severe detriments in production and reuptake of DA as manifested by reduced striatal $\mathrm{TH}$ and DAT proteins, respectively (Fig. 3). These findings suggest that the defective STR and its connected circuitry are major features of ASD. Furthermore, the susceptibility of striatal $\mathrm{TH}$ protein to intranasal DA treatment in BTBR and Fmr1-KO mice (Fig. 4) indicate that the STR can be an effective target for therapeutic interventions of ASD. Our results provide not only empirical evidence for the DA hypothesis of ASD [80], but also a proof of principle for developing clinical treatments for the disorder.

\footnotetext{
Abbreviations

ASD: Autistic spectrum disorder; FXS: Fragile X syndrome; ADHD: Attentiondeficit/hyperactivity disorder; BTBR: Black and Tan BRachyury $T^{+} \mid \mathrm{tp} 3^{\mathrm{tf}} / \mathrm{J}$; Fmr 1 KO: Fragile X Mental Retardation 1 knockout; WT: Wild type; DA: Dopamine; TH: Tyrosine hydroxylase; DAT: Dopamine transporter; VGLUT1: Vesicular glutamate transporter 1; GAD67: Glutamic acid decarboxylase 67; SNc: Substantia nigra pars compacta; VTA: Ventral tegmental area;
} 
STR: Striatum; dSTR: Dorsal striatum; NAc: Nucleus accumbens; ROI: Region of interest; Db: Box-counting dimension; SEM: Standard error of mean

\section{Acknowledgements}

No additional acknowledgements.

\section{Authors' contributions}

YMY and JPH designed the project. OYC performed immunohistochemistry and behavioral analyses with the assistance of HZ, ND, JSL, and CM. SSP conducted Western blot experiments. SN provided critical inputs. OYC, SN, JPH and YMY wrote the manuscript. The authors read and approved the final manuscript.

\section{Funding}

This work was supported by the National Institute of Neurological Disorders and Stroke (NINDS) of the National Institutes of Health (NIH) grant R15NS112964 to YMY, the University of Minnesota start-up fund to YMY, and the German National Science Foundation (DFG) grant HU 306/27-3 to JPH.

\section{Availability of data and materials}

All the data are available upon request.

\section{Ethics approval and consent to participate}

Not applicable.

\section{Consent for publication}

Not applicable.

\section{Competing interests}

The authors declare no competing financial and non-financial interests in relation to this work. CM is an employee of $M$ et P Pharma AG. However, the company did not play a role in any aspect of the study.

\section{Author details}

${ }^{1}$ Department of Biomedical Sciences, University of Minnesota Medical School, 1035 University Drive, Duluth, MN 55812, USA. ²Department of Psychology, National Chung Cheng University, Minhsiung, Chiayi, Taiwan, Republic of China. ${ }^{3} \mathrm{M}$ et $\mathrm{P}$ Pharma AG, Emmetten, Switzerland. ${ }^{4}$ Oceanographic Center, Nova Southeastern University, Fort Lauderdale, FL 33314, USA. ${ }^{5}$ Clinic of Nuclear Medicine, Heinrich Heine University of Düsseldorf, Düsseldorf, Germany. ${ }^{6}$ Center for Behavioral Neuroscience, Heinrich Heine University of Düsseldorf, Universitaetsstr. 1, 40225 Düsseldorf, Germany. ${ }^{7}$ Department of Neuroscience, University of Minnesota, Minneapolis, MN 55455, USA.

Received: 29 May 2020 Accepted: 30 July 2020

Published online: 10 August 2020

\section{References}

1. Lai MC, Lombardo MV, Baron-Cohen S. Autism. Lancet. 2014;383(9920):896910.

2. Simonoff E, Pickles A, Charman T, Chandler S, Loucas T, Baird G. Psychiatric disorders in children with autism spectrum disorders: prevalence, comorbidity, and associated factors in a population-derived sample. J Am Acad Child Adolesc Psychiatry. 2008;47(8):921-9.

3. Gandal MJ, Haney JR, Parikshak NN, Leppa V, Ramaswami G, Hartl C, Schork AJ, Appadurai V, Buil A, Werge TM, et al. Shared molecular neuropathology across major psychiatric disorders parallels polygenic overlap. Science. 2018; 359(6376):693-7

4. Bromberg-Martin ES, Matsumoto M, Hikosaka O. Dopamine in motivational control: rewarding, aversive, and alerting. Neuron. 2010;68(5):815-34.

5. Volkow ND, Wang GJ, Newcorn J, Telang F, Solanto MV, Fowler JS, Logan J, Ma Y, Schulz K, Pradhan K, et al. Depressed dopamine activity in caudate and preliminary evidence of limbic involvement in adults with attentiondeficit/hyperactivity disorder. Arch Gen Psychiatry. 2007:64(8):932-40.

6. Cousins DA, Butts $\mathrm{K}$, Young AH. The role of dopamine in bipolar disorder. Bipolar Disord. 2009;11(8):787-806.

7. Harrison PJ, Weinberger DR. Schizophrenia genes, gene expression, and neuropathology: on the matter of their convergence. Mol Psychiatry. 2005; 10(1):40-68 image 45.

8. Hamilton PJ, Campbell NG, Sharma S, Erreger K, Herborg Hansen F, Saunders C, Belovich AN, Consortium NAAS, Sahai MA, Cook EH, et al. De novo mutation in the dopamine transporter gene associates dopamine dysfunction with autism spectrum disorder. Mol Psychiatry. 2013;18(12): 1315-23.

9. Hollander E, Anagnostou E, Chaplin W, Esposito K, Haznedar MM, Licalzi E, Wasserman S, Soorya L, Buchsbaum M. Striatal volume on magnetic resonance imaging and repetitive behaviors in autism. Biol Psychiatry. 2005; 58(3):226-32.

10. Langen M, Schnack HG, Nederveen $H$, Bos D, Lahuis BE, de Jonge MV, van Engeland $\mathrm{H}$, Durston S. Changes in the developmental trajectories of striatum in autism. Biol Psychiatry. 2009;66(4):327-33.

11. Fuccillo MV. Striatal circuits as a common node for autism pathophysiology. Front Neurosci. 2016;10:27.

12. Patterson PH. Modeling autistic features in animals. Pediatr Res. 2011;69(5 Pt 2):34R-40R

13. Zurcher NR, Bhanot A, McDougle CJ, Hooker JM. A systematic review of molecular imaging (PET and SPECT) in autism spectrum disorder: current state and future research opportunities. Neurosci Biobehav Rev. 2015:52:56-73.

14. Jobski K, Hofer J, Hoffmann F, Bachmann C. Use of psychotropic drugs in patients with autism spectrum disorders: a systematic review. Acta Psychiatr Scand. 2017;135(1):8-28

15. McFarlane HG, Kusek GK, Yang M, Phoenix JL, Bolivar VJ, Crawley JN Autism-like behavioral phenotypes in BTBR T+tf/J mice. Genes Brain Behav. 2008;7(2):152-63.

16. Chao OY, Yunger R, Yang YM. Behavioral assessments of BTBR T+Itpr3tf/J mice by tests of object attention and elevated open platform: implications for an animal model of psychiatric comorbidity in autism. Behav Brain Res. 2018:347:140-7.

17. Chao OY, Marron Fernandez de Velasco E, Pathak SS, Maitra S, Zhang H, Duvick L, Wickman K, Orr HT, Hirai H, Yang YM. Targeting inhibitory cerebellar circuitry to alleviate behavioral deficits in a mouse model for studying idiopathic autism. Neuropsychopharmacology. 2020;45:1159-70. PMID: 32179875.

18. Dodero L, Damiano M, Galbusera A, Bifone A, Tsaftsaris SA, Scattoni ML, Gozzi A. Neuroimaging evidence of major morpho-anatomical and functional abnormalities in the BTBR T+TF/J mouse model of autism. PLoS One. 2013;8(10):e76655.

19. Ellegood J, Babineau BA, Henkelman RM, Lerch JP, Crawley JN. Neuroanatomical analysis of the BTBR mouse model of autism using magnetic resonance imaging and diffusion tensor imaging. Neuroimage. 2013;70:288-300.

20. Squillace M, Dodero L, Federici M, Migliarini S, Errico F, Napolitano F, Krashia P, Di Maio A, Galbusera A, Bifone A, et al. Dysfunctional dopaminergic neurotransmission in asocial BTBR mice. Transl Psychiatry. 2014;4:e427.

21. Bakker CE, Verheij C, Wilemsen R, van der Helm R, Oerlemans F, Vermey $M$, Bygrave A, Hoogeveen A, Oostra BA, Reyniers E, et al. Fmr1 knockout mice: a model to study fragile X mental retardation. Cell. 1994;78(1):23-33.

22. Harris SW, Hessl D, Goodlin-Jones B, Ferranti J, Bacalman S, Barbato I, Tassone F, Hagerman PJ, Herman H, Hagerman RJ. Autism profiles of males with fragile X syndrome. Am J Ment Retard. 2008;113(6):427-38.

23. Fish EW, Krouse MC, Stringfield SJ, Diberto JF, Robinson JE, Malanga CJ. Changes in sensitivity of reward and motor behavior to dopaminergic, glutamatergic, and cholinergic drugs in a mouse model of fragile $X$ syndrome. PLoS One. 2013;8(10):e77896.

24. Fulks JL, O'Bryhim BE, Wenzel SK, Fowler SC, Vorontsova E, Pinkston JW, Ortiz AN, Johnson MA. Dopamine release and uptake impairments and behavioral alterations observed in mice that model fragile $X$ mental retardation syndrome. ACS Chem Neurosci. 2010;1(10):679-90.

25. Wang H, Wu LJ, Kim SS, Lee FJ, Gong B, Toyoda H, Ren M, Shang YZ, Xu H, Liu $F$, et al. FMRP acts as a key messenger for dopamine modulation in the forebrain. Neuron. 2008;59(4):634-47.

26. Paul K, Venkitaramani DV, Cox CL. Dampened dopamine-mediated neuromodulation in prefrontal cortex of fragile X mice. J Physiol. 2013; 591(4):1133-43.

27. Meyza KZ, Blanchard DC. The BTBR mouse model of idiopathic autism Current view on mechanisms. Neurosci Biobehav Rev. 2017;76(Pt A):99-110.

28. Baio J, Wiggins L, Christensen DL, Maenner MJ, Daniels J, Warren Z, KurziusSpencer M, Zahorodny W, Robinson Rosenberg C, White T, et al. Prevalence of autism Spectrum disorder among children aged 8 years - autism and developmental disabilities monitoring network, 11 sites, United States, 2014. MMWR Surveill Summ. 2018:67(6):1-23.

29. Franklin KBJ, Paxinos $\mathrm{G}$. The mouse brain in stereotaxic coordinates, compact third edition. 3rd ed: Academic Press; 2008. ISBN: 9780123742445. ISBN: 9780080921365. 
30. Jelinek HF, Fernandez E. Neurons and fractals: how reliable and useful are calculations of fractal dimensions? J Neurosci Methods. 1998;81(1-2):9-18.

31. Rajkovic N, Krstonosic B, Milosevic N. Box-counting method of 2D neuronal image: method modification and quantitative analysis demonstrated on images from the monkey and human brain. Comput Math Methods Med. 2017;2017:8967902.

32. Lee C. Protein extraction from mammalian tissues. Methods Mol Biol. 2007; 362:385-9.

33. Yang YM, Arsenault J, Bah A, Krzeminski M, Fekete A, Chao OY, Pacey LK, Wang A, Forman-Kay J, Hampson DR, et al. Identification of a molecular locus for normalizing dysregulated GABA release from interneurons in the fragile X brain. Mol Psychiatry. 2018. https://doi.org/10.1038/s41380-0180240-0.

34. Kholodar A, Amikishieva A, Anisimov M. Effects of intranasal administration of dopamine on anxiety and locomotor activity in two mouse strains. Neurosci Behav Physiol. 2013;43:409-15.

35. Walf AA, Frye CA. The use of the elevated plus maze as an assay of anxietyrelated behavior in rodents. Nat Protoc. 2007;2(2):322-8.

36. Aspide R, Gironi Carnevale UA, Sergeant JA, Sadile AG. Non-selective attention and nitric oxide in putative animal models of attention-deficit hyperactivity disorder. Behav Brain Res. 1998;95(1):123-33.

37. Kalueff AV, Stewart AM, Song C, Berridge KC, Graybiel AM, Fentress JC. Neurobiology of rodent self-grooming and its value for translational neuroscience. Nat Rev Neurosci. 2016;17(1):45-59.

38. Milani H, Schwarting RK, Kumpf S, Steiner H, Huston JP. Interaction between recovery from behavioral asymmetries induced by hemivibrissotomy in the rat and the effects of apomorphine and amphetamine. Behav Neurosci. 1990;104(3):470-6.

39. Simon $P$, Dupuis $R$, Costentin J. Thigmotaxis as an index of anxiety in mice. Influence of dopaminergic transmissions. Behav Brain Res. 1994;61(1):59-64.

40. Prut $L$, Belzung $C$. The open field as a paradigm to measure the effects of drugs on anxiety-like behaviors: a review. Eur J Pharmacol. 2003;463(1-3):3-33.

41. Silverman JL, Yang M, Lord C, Crawley JN. Behavioural phenotyping assays for mouse models of autism. Nat Rev Neurosci. 2010;11(7):490-502.

42. Fremeau RT Jr, Troyer MD, Pahner I, Nygaard GO, Tran CH, Reimer RJ, Bellocchio EE, Fortin D, Storm-Mathisen J, Edwards RH. The expression of vesicular glutamate transporters defines two classes of excitatory synapse. Neuron. 2001;31(2):247-60.

43. Fujiyama F, Unzai T, Nakamura K, Nomura S, Kaneko T. Difference in organization of corticostriatal and thalamostriatal synapses between patch and matrix compartments of rat neostriatum. Eur J Neurosci. 2006;24(10): 2813-24.

44. Moss J, Bolam JP. A dopaminergic axon lattice in the striatum and its relationship with cortical and thalamic terminals. J Neurosci. 2008;28(44): $11221-30$.

45. Kreitzer AC. Physiology and pharmacology of striatal neurons. Annu Rev Neurosci. 2009:32:127-47.

46. Dhuria SV, Hanson LR, Frey WH 2nd. Intranasal delivery to the central nervous system: mechanisms and experimental considerations. J Pharm Sci. 2010;99(4):1654-73.

47. Ramos A. Animal models of anxiety: do I need multiple tests? Trends Pharmacol Sci. 2008;29(10):493-8.

48. Ventura R, Pascucci T, Catania MV, Musumeci SA, Puglisi-Allegra S. Object recognition impairment in Fmr1 knockout mice is reversed by amphetamine: involvement of dopamine in the medial prefrontal cortex. Behav Pharmacol. 2004;15(5-6):433-42.

49. Sorensen EM, Bertelsen F, Weikop P, Skovborg MM, Banke T, Drasbek KR, ScheelKruger J. Hyperactivity and lack of social discrimination in the adolescent Fmr1 knockout mouse. Behav Pharmacol. 2015; 26(8 Spec No):733-740.

50. Martin L, Sample H, Gregg M, Wood C. Validation of operant social motivation paradigms using BTBR T+tf/J and C57BL/6J inbred mouse strains. Brain Behav. 2014;4(5):754-64.

51. Pearson BL, Bettis JK, Meyza KZ, Yamamoto LY, Blanchard DC, Blanchard RJ. Absence of social conditioned place preference in BTBR T+tf/J mice: relevance for social motivation testing in rodent models of autism. Behav Brain Res. 2012;233(1):99-104.

52. Bagni C, Zukin RS. A synaptic perspective of fragile $X$ syndrome and autism spectrum disorders. Neuron. 2019;101(6):1070-88.

53. Morales J, Hiesinger PR, Schroeder AJ, Kume K, Verstreken P, Jackson FR, Nelson DL, Hassan BA. Drosophila fragile $X$ protein, DFXR, regulates neuronal morphology and function in the brain. Neuron. 2002;34(6):961-72.
54. Dockendorff TC, Su HS, McBride SM, Yang Z, Choi CH, Siwicki KK, Sehgal A, Jongens TA. Drosophila lacking dfmr1 activity show defects in circadian output and fail to maintain courtship interest. Neuron. 2002;34(6):973-84.

55. Pan L, Zhang YQ, Woodruff E, Broadie K. The Drosophila fragile X gene negatively regulates neuronal elaboration and synaptic differentiation. Curr Biol. 2004;14(20):1863-70.

56. Zimmer SE, Doll SG, Garcia ADR, Akins MR. Splice form-dependent regulation of axonal arbor complexity by FMRP. Dev Neurobiol. 2017;77(6):738-52.

57. La Fata G, Gartner A, Dominguez-Iturza N, Dresselaers T, Dawitz J, Poorthuis RB, Averna M, Himmelreich U, Meredith RM, Achsel T, et al. FMRP regulates multipolar to bipolar transition affecting neuronal migration and cortical circuitry. Nat Neurosci. 2014;17(12):1693-700.

58. Sandoval GM, Shim S, Hong DS, Garrett AS, Quintin EM, Marzelli MJ, Patnaik S, Lightbody AA, Reiss AL. Neuroanatomical abnormalities in fragile $X$ syndrome during the adolescent and young adult years. J Psychiatr Res. 2018;107:138-44.

59. Giampetruzzi A, Carson JH, Barbarese E. FMRP and myelin protein expression in oligodendrocytes. Mol Cell Neurosci. 2013;56:333-41.

60. Pacey LK, Xuan IC, Guan S, Sussman D, Henkelman RM, Chen Y, Thomsen C, Hampson DR. Delayed myelination in a mouse model of fragile $X$ syndrome. Hum Mol Genet. 2013;22(19):3920-30.

61. Gholizadeh S, Halder SK, Hampson DR. Expression of fragile X mental retardation protein in neurons and glia of the developing and adult mouse brain. Brain Res. 2015;1596:22-30.

62. Surmeier DJ, Ding J, Day M, Wang Z, Shen W. D1 and D2 dopaminereceptor modulation of striatal glutamatergic signaling in striatal medium spiny neurons. Trends Neurosci. 2007;30(5):228-35.

63. Giros B, Jaber M, Jones SR, Wightman RM, Caron MG. Hyperlocomotion and indifference to cocaine and amphetamine in mice lacking the dopamine transporter. Nature. 1996;379(6566):606-12.

64. Silverman JL, Babineau BA, Oliver CF, Karras MN, Crawley JN. Influence of stimulant-induced hyperactivity on social approach in the BTBR mouse model of autism. Neuropharmacology. 2013;68:210-22.

65. de Souza Silva MA, Topic B, Huston JP, Mattern C. Intranasal dopamine application increases dopaminergic activity in the neostriatum and nucleus accumbens and enhances motor activity in the open field. Synapse. 2008;62(3):176-84.

66. Janenaite $E$, Vengeliene $V$, Bespalov A, Behl B. Potential role of tyrosine hydroxylase in the loss of psychostimulant effect of amphetamine under conditions of impaired dopamine transporter activity. Behav Brain Res. 2017; 334:105-8.

67. Khlghatyan J, Beaulieu JM. Are FXR family proteins integrators of dopamine signaling and glutamatergic neurotransmission in mental illnesses? Front Synaptic Neurosci. 2018;10:22.

68. Ruocco LA, Treno C, Gironi Carnevale UA, Arra C, Mattern C, Huston JP, de Souza Silva MA, Nikolaus S, Scorziello A, Nieddu M, et al. Prepuberal intranasal dopamine treatment in an animal model of ADHD ameliorates deficient spatial attention, working memory, amino acid transmitters and synaptic markers in prefrontal cortex, ventral and dorsal striatum. Amino Acids. 2014:46(9):2105-22.

69. de Souza Silva MA, Mattern C, Decheva C, Huston JP, Sadile AG, Beu M, Muller HW, Nikolaus S. Intranasal dopamine reduces in vivo [(123) I]FP-CIT binding to striatal dopamine transporter: correlation with behavioral changes and evidence for Pavlovian conditioned dopamine response. Front Behav Neurosci. 2016;10:80.

70. Dahlin M, Jansson B, Bjork E. Levels of dopamine in blood and brain following nasal administration to rats. Eur J Pharm Sci. 2001;14(1):75-80.

71. Buddenberg TE, Topic B, Mahlberg ED, de Souza Silva MA, Huston JP, Mattern C. Behavioral actions of intranasal application of dopamine: effects on forced swimming, elevated plus-maze and open field parameters. Neuropsychobiology. 2008;57(1-2):70-9.

72. Talbot T, Mattern C, de Souza Silva MA, Brandao ML. Intranasal administration of dopamine attenuates unconditioned fear in that it reduces restraint-induced ultrasound vocalizations and escape from bright light. J Psychopharmacol. 2017:31(6):682-90.

73. Pum ME, Schable S, Harooni HE, Topic B, De Souza Silva MA, Li JS, Huston JP, Mattern C. Effects of intranasally applied dopamine on behavioral asymmetries in rats with unilateral 6-hydroxydopamine lesions of the nigrostriatal tract. Neuroscience. 2009;162(1):174-83.

74. Trossbach SV, de Souza Silva MA, Huston JP, Korth C, Mattern C. Intranasal dopamine treatment reinstates object-place memory in aged rats. Neurobiol Learn Mem. 2014;114:231-5. 
75. Wang AL, Chao OY, Yang YM, Trossbach SV, Muller CP, Korth C, Huston JP, de Souza Silva MA. Anxiogenic-like behavior and deficient attention/ working memory in rats expressing the human DISC1 gene. Pharmacol Biochem Behav. 2019:179:73-9.

76. Ruocco LA, de Souza Silva MA, Topic B, Mattern C, Huston JP, Sadile AG. Intranasal application of dopamine reduces activity and improves attention in Naples high excitability rats that feature the mesocortical variant of ADHD. Eur Neuropsychopharmacol. 2009;19(10):693-701.

77. Peca J, Feliciano C, Ting JT, Wang W, Wells MF, Venkatraman TN, Lascola CD, Fu Z, Feng G. Shank3 mutant mice display autistic-like behaviours and striatal dysfunction. Nature. 2011;472(7344):437-42.

78. Langen M, Leemans A, Johnston P, Ecker C, Daly E, Murphy CM, Dell'acqua F, Durston S, Consortium A, Murphy DG. Fronto-striatal circuitry and inhibitory control in autism: findings from diffusion tensor imaging tractography. Cortex. 2012;48(2):183-93.

79. Delmonte S, Gallagher L, O'Hanlon E, McGrath J, Balsters JH. Functional and structural connectivity of frontostriatal circuitry in autism spectrum disorder. Front Hum Neurosci. 2013;7:430.

80. Paval D. A dopamine hypothesis of autism spectrum disorder. Dev Neurosci. 2017;39(5):355-60

\section{Publisher's Note}

Springer Nature remains neutral with regard to jurisdictional claims in published maps and institutional affiliations.

Ready to submit your research? Choose BMC and benefit from:

- fast, convenient online submission

- thorough peer review by experienced researchers in your field

- rapid publication on acceptance

- support for research data, including large and complex data types

- gold Open Access which fosters wider collaboration and increased citations

- maximum visibility for your research: over $100 \mathrm{M}$ website views per year

At $\mathrm{BMC}$, research is always in progress.

Learn more biomedcentral.com/submissions 